Notfall Rettungsmed 2019 $\cdot 22: 334-338$ https://doi.org/10.1007/s10049-019-0609-x Online publiziert:27. Mai 2019

(c) Springer Medizin Verlag GmbH, ein Teil von Springer Nature 2019

\section{Zusatzmaterial online}

Die Online-Version dieses Beitrags (https:// doi.org/10.1007/s10049-019-0609-x) enthält das vollständige Positionspapier des Europäischen Rats für Wiederbelebung. Beitrag und Zusatzmaterial stehen Ihnen im elektronischen Volltextarchiv auf http:// www.springermedizin.de/notfall-undrettungsmedizin zur Verfügung. Sie finden das Zusatzmaterial am Beitragsende unter "Supplementary Material".
Dieser Beitrag wurde für Editors Pick ausgewählt und ist damit frei zugänglich.

B. Dirks ${ }^{1} \cdot$ S. Wingen ${ }^{2} \cdot$ G. Rücker ${ }^{3} \cdot$ R. Greif ${ }^{4} \cdot$ H. Papaspyrou ${ }^{5} \cdot$ B. W. Böttiger ${ }^{2}$

${ }^{1}$ GRC Geschäftsstelle, Sektion Notfallmedizin, Universitätsklinikum Ulm, Ulm, Deutschland

${ }^{2}$ Klinik für Anästhesiologie und Operative Intensivmedizin, Universitätsklinikum Köln (AöR), Köln, Deutschland

${ }^{3}$ Klinik und Poliklinik für Anästhesiologie und Intensivtherapie, Rostocker Simulationsanlage und Notfallausbildungszentrum (RoSaNa), Universitätsmedizin Rostock, Rostock, Deutschland

${ }^{4}$ Dept. Anesthesiology and Pain Therapy, Bern University Hospital, University of Bern, Bern, Schweiz

${ }^{5}$ Abteilung für Anästhesie und Intensivmedizin, Klinikum Stadt Soest, Soest, Deutschland

\title{
Modularer Lehrerausbildungs- kurs des Deutschen Rates für Wiederbelebung (GRC) für den Wiederbelebungsunterricht in Schulen
}

\section{Positionspapier des GRC nach der Empfehlung der Deutschen Kultusministerkonferenz und dem aktuellen GRC-Mustercurriculum}

\section{Hintergrund und Bedeutung des modularen GRC- Lehrerausbildungskurses}

Der Herz-Kreislauf-Stillstand stellt den Dringlichsten aller Notfälle und gleichzeitig die dritthäufigste Todesursache dar. Wenn der Herzschlag ausfällt, wird das Gehirn nicht mehr mit Sauerstoff versorgt. In der Folge gehen Gehirnzellen zugrunde, die nicht mehr wiederhergestellt werden können. Im Normalfall, also bei Gesunden, hält der Sauerstoffvorrat noch 3-5 Minuten vor, bei Vorerkrankten oftmals weniger. Der Rettungsdienst benötigt jedoch durchschnittlich 8 und mehr Minuten bis zum Eintreffen beim Patienten, da die Rettungswachen regional gleichmäßig verteilt sind und die Fahrt und das Aufsuchen des Notfallortes Zeit in Anspruch nimmt. Zwischen dem Herz-Kreislauf-Stillstand und dem Eintreffen des professionellen Rettungssystems liegen also mehrere Minuten, in denen ohne Wiederbelebungsmaßnah- men das Gehirn nicht zurückführbare Schädigungen davon trägt.

Glücklicherweise kann die Zeit bis zum Eintreffen des Rettungsdienstes überbrückt und das Gehirn weiter mit Sauerstoff versorgt werden, wenn sofort Wiederbelebungsmaßnahmen durch anwesende Laien eingeleitet werden. Die hierfür erforderlichen Maßnahmen sind selbst von Kindern einfach zu erlernen. Ihre Anwendung rettet unmittelbar Menschen vor dem Tod. Zahlreiche Menschen verdanken den beherzten Laienhelfern ihr Leben (,Wiederbelebung ist kinderleicht").

Vor diesem Hintergrund hat im Jahr 2014 die Konferenz der Kultusminister der Länder in der Bundesrepublik Deutschland die Implementierung des Wiederbelebungsunterrichtes in Schulen bundesweit empfohlen. Gemäß Beschluss sollen Schülerinnen und Schüler ab der 7. Klasse jährlich im Umfang von zwei Unterrichtsstunden in den lebens- 
Tab. 1 Zeitplan des modularen GRC-Lehrerausbildungskurses

\begin{tabular}{|c|c|c|}
\hline Dauer & Modul & Inhalt \\
\hline 00:00 & Beginn & Begrüßung \\
\hline 00:05 & $\begin{array}{l}\text { Einführung ins } \\
\text { Thema }\end{array}$ & Erfolgreich wiederbeleben und das Konzept „KIDS SAVE LIVES“ \\
\hline 00:25 & $\begin{array}{l}\text { Konzept- } \\
\text { vorstellung }\end{array}$ & 1. Ebene: Prüfen, Rufen, Drücken \\
\hline 00:40 & $\begin{array}{l}\text { CPR-Unterricht: } \\
\text { Basis mit Praxis }\end{array}$ & $\begin{array}{l}\text { Unterricht von kardiopulmonaler Reanimation (CPR) in } 4 \text { Schrit- } \\
\text { ten: } \\
\text { Schritt 1: Demonstration in Echtzeit } \\
\text { Schritt 2: Demonstration mit Kommentaren } \\
\text { Schritt 3: Teilnehmer leitet Ausbilder an } \\
\text { Schritt 4: Teilnehmer üben CPR } \\
\text { Die Lernziele sind: } \\
\text { Lehrerinnen und Lehrer lernen in kurzer Zeit, wie CPR effektiv } \\
\text { und richtig erlernt, unterrichtet und praktiziert werden kann. } \\
\text { Dies kann in 20-30 min. erreicht werden. } \\
\text { Danach wird auf die Schlüsselpunkte eingegangen, die den Un- } \\
\text { terricht von BLS-Kompetenzen erleichtern. Beispielhaft werden } \\
\text { Erfahrungen vorgestellt, an welcher Stelle Vermittlungsprobleme } \\
\text { auftreten können. } \\
\text { Die Lehrerinnen und Lehrer bekommen die Möglichkeit, den } \\
\text { Unterricht von CPR zu reflektieren. Dabei helfen Beispiele zur } \\
\text { Fehlerkorrektur. Das kann u. a. anhand von Unterrichtsszenarien } \\
\text { mit Feedback oder Nachbesprechung erreicht werden }\end{array}$ \\
\hline 01:45 & Pause & - \\
\hline 02:00 & $\begin{array}{l}\text { Konzept- } \\
\text { vorstellung }\end{array}$ & 2. Ebene: Beatmung + AED im CPR-Unterricht \\
\hline 02:10 & $\begin{array}{l}\text { CPR Unterricht: } \\
\text { Erweitert mit } \\
\text { Beatmung + AED } \\
\text { inklusive Praxis }\end{array}$ & $\begin{array}{l}\text { Erweiterter CPR-Unterricht: Beatmung/AED wieder in den } \\
4 \text { Schritten: } \\
\text { Schritt 1: Echtzeitdemo } \\
\text { Schritt 2: Instruktor kommentiert Kompetenzen } \\
\text { Schritt 3: Anleitung des Instruktors durch die Teilnehmer } \\
\text { Schritt 4: Teilnehmerübung mit Unterrichtfokus auf Hinweise, wie } \\
\text { nicht richtige Handlungen verbessert werden können } \\
\text { Diese Unterrichtseinheit hat als Lernziele: } \\
\text { Korrektes Prüfen, Rufen, Drücken, Beatmung, AED anbringen, } \\
\text { Defibrillation (einmalig und erfolgreich) } \\
\text { Dies wird anhand von Fallbeispielen erreicht: } \\
\text { - Einmalige erfolgreiche Defibrillation (analog zur Demonstrati- } \\
\text { on) } \\
\text { - Kein Schock erforderlich (jedoch CPR erforderlich) } \\
\text { - Anhaltender defibrillationspflichtiger Rhythmus (2 Defibrilla- } \\
\text { tionen) } \\
\text { CPR-Fertigkeiten werden auf korrekte Durchführung überprüft } \\
\text { und gegebenenfalls korrigiert. } \\
\text { Endziel ist der Unterricht von einer sicheren und effektiven CPR }\end{array}$ \\
\hline 03:25 & Kursabschluss & $\begin{array}{l}\text { GRC-Lehrkonzept: } \\
\text { Motivatoren, Hindernisse, Ängste im CPR Unterricht } \\
\text { Lehrmaterialen für den CPR-Unterricht in Schulen } \\
\text { Rechtliche Aspekte von der CPR }\end{array}$ \\
\hline 03:40 & $\begin{array}{l}\text { CPR Praxis } \\
\text { Ende }\end{array}$ & $\begin{array}{l}\text { Gezieltes Üben der erlernten Unterrichtskompetenzen } \\
\text { Zusammenfassung des Kurses und Feedback der Teilnehmenden, } \\
\text { ggf. Evaluation }\end{array}$ \\
\hline \multicolumn{3}{|c|}{$\begin{array}{l}\text { CPR kardiopulmonale Reanimation (Herz-Lungen-Wiederbelebung), AED Automatisierter Externer } \\
\text { Defibrillator }\end{array}$} \\
\hline
\end{tabular}

rettenden Wiederbelebungsmaßnahmen unterrichtet werden.

Lehrerinnen und Lehrer sind in gleicher Weise wie medizinisches Fachpersonal in der Lage, eine Wiederbelebung erfolgreich durchzuführen und Schülerinnen und Schülern die Grundbegriffe der Wiederbelebung beizubringen. Die vorliegende Fortbildung soll in klar strukturierten Schritten Lehrerinnen und Lehrer dazu befähigen, Wiederbelebungsunterricht in Schulen selbstständig durchzuführen. Ein Ziel ist es, die derzeit vergleichsweise niedrige Laienwiederbelebungsrate landesweit weiter und nachhaltig zu verbessern und dadurch 10.000 Menschenleben zusätzlich pro Jahr in Deutschland zu retten. Gleichzeitig kann so auch die Sozialkompetenz der Schülerinnen und Schüler gefördert werden.

Das globale Ziel dieses Wiederbelebungsunterrichtes in Schulen ist es, die Basismaßnahmen der Herz-LungenWiederbelebung (Basic Life Support, BLS) rasch und sicher jederzeit durchführen zu können. Somit soll mittelfristig jede Bürgerin und jeder Bürger mit den Grundkompetenzen zur Wiederbelebung vertraut gemacht werden, um so das Überleben der Betroffenen deutlich zu verbessern.

Der weitere Hintergrund zum Wiederbelebungsunterricht in Schulen wird sehr anschaulich im Positionspapiers des Europäischen Rats für Wiederbelebung beschrieben, das Ihnen online als Beitrag zur Verfügung gestellt wird (deutsche Übersetzung des GRC unter www. grc-org.de) [1].

Die darin enthaltenen 10 Thesen zu „KIDS SAVE LIVES“ veranschaulichen die Grundlage und die Wichtigkeit sowie die praktische Umsetzung der Ausbildung von Schülerinnen und Schülern in Wiederbelebung.

\section{Kurszielsetzung}

Der Deutsche Rat für Wiederbelebung hat, basierend auf den internationalen Empfehlungen zur Herz-Lungen-Wiederbelebung und auf eigenen umfangreichen Erfahrungen und Kurskonzepten, zur Ausbildung in Wiederbelebung gemeinsam mit zahlreichen Hilfsorganisationen einen bereits mehrfach überprüf- 
ten modularen GRC-Lehrerausbildungskurs für den Wiederbelebungsunterricht in Schulen entwickelt.

Ziel ist es, Lehrerinnen und Lehrer durch diesen Kurs unmittelbar, nachhaltig und sicher in die Lage zu versetzen, autark die einfachen, grundlegenden und lebensrettenden Wiederbelebungsmaßnahmen erfolgreich an Schülerinnen und Schüler weiterzugeben.

Nach erfolgreicher Absolvierung des modularen GRC-Lehrerausbildungskurses können die Lehrerinnen und Lehrer ihren Schülerinnen und Schülern sowohl die einfachen Basismaßnahmen der Wiederbelebung (PRÜFEN - RUFEN DRÜCKEN) als auch die erweiterten Basismaßnahmen (Beatmung - Einsatz eines Defibrillators) effektiv und nachhaltig beibringen.

Darüber hinaus zielt der modulare GRC-Lehrerausbildungskurs darauf $a b$, den Lehrerinnen und Lehrern umfangreiche Unterrichtsmaterialien an die Hand zu geben, mit denen sie die Implementierung des Wiederbelebungsunterrichtes in der eigenen Schule ganz einfach durchführen können.

\section{Kursinhalte und Zeitplan}

Der modulare GRC-Lehrerausbildungskurs ist ein insgesamt vierstündiger Kurs, der folgende Module beinhaltet:

- Das Erkennen eines Kreislaufstillstandes („Prüfen“)

- Die Alarmierung des Rettungsdienstes („Rufen“)

- Das Erlernen der Herzdruckmassage/ Thoraxkompressionen („Drücken“)

- Die Atemspende (Mund-zu-MundBeatmung)

- Die Verwendung eines automatisierten externen Defibrillators (AED)

Diese einzelnen Ausbildungsschritte sind nach den bisher vorliegenden Studien und Erfahrungen für die folgenden Altersstufen zu empfehlen:

- Ab dem 4. Lebensjahr (Kindergarten): Das Erkennen eines Kreislaufstillstandes („Prüfen“) und die Alarmierung des Rettungsdienstes („Rufen“)
- Ab dem 12.-14. Lebensjahr (Schule): Das Erlernen der Herzdruckmassage („Drücken")

- Ab dem 14. Lebensjahr: Die Atemspende (Mund-zu-Mund Beatmung) sowie darauf aufbauend die Verwendung eines AED

Es wird empfohlen, Schülerinnen und Schüler jährlich mit einer Doppelstunde entsprechend auszubilden und dies so lange zu wiederholen, so lange sie in eine Schule gehen. Die zugrunde liegende Evidenz zu dieser Empfehlung, die sich auch in dem Beschluss der Kultusministerkonferenz widerspiegelt, kommt aus Studien zu „spaced learning“, d.h. kurze Einheiten und so oft wie möglich. Diese kurzen Einheiten sind erfolgreicher als längere Kurse am Stück. Somit folgt das vorliegende Konzept modernen didaktischen Prinzipien.

In - Tab. 1 wird ein Überblick über den Zeitplan des modularen GRC-Lehrerausbildungskurses gegeben.

Zur Vermittlung der Wiederbelebungsfertigkeiten eignet sich nach der Erfahrung des GRC besonders ein schrittweiser Zugang, um die Lernziele zu erreichen. Zentral wichtig sind bei diesem schrittweisen Lernen das praktische Üben mit korrigierenden und positiv verstärkenden Rückmeldung zur Ausführung sowie das häufige Wiederholen.

Die vorgeschlagenen vier Schritte im Kurskonzept sind:

1. Die Demonstration der Maßnahmen in Echtzeit ohne zusätzliche Erklärungen. Damit soll gezeigt werden, was erwartet wird, wie das richtig gemacht wird und was am Ende des Kurses von den am Unterricht Teilnehmenden erwartet wird. Dazu gehören: Das Ansprechen der betroffenen Person, der Hilferuf und Hilfe holen, der Kontakt mit der Rettungsleitstelle und die korrekt durchgeführten Erstmaßnahmen.

2. Nun demonstriert der Instruktor alle Maßnahmen mit seinen Kommentaren, erklärt den Hintergrund zur Durchführung einzelner Teilschritte und liefert notwendige theoretische Erklärungen, die das Verständnis und das Behalten der Einzelschritte fördern können.

3. In diesem Schritt leitet ein Teilnehmer die Maßnahmen an und der Instruktor führt diese aus, wenn sie korrekt angeleitet worden sind ${ }^{1}$. Ziel ist es dabei, zu überprüfen, ob unterrichtete Inhalte richtig verstanden wurden und kognitiv umgesetzt werden können. Zudem kann der unterrichtende Instruktor überprüfen, ob sein Unterricht geeignet war, das zu vermitteln, was beabsichtigt war.

4. Das gründliche Üben der Maßnahmen durch die Teilnehmer (mit Feedback zu deren Performance durch den Ausbilder) ist wichtig, um die vorgegebenen Lernziele sicher zu erreichen. In diesem Schritt korrigiert der Ausbilder alle Fehler der Teilnehmenden und verstärkt richtige Maßnahmen und korrektes Verhalten. Peerfeedback - also Feedback auch durch die Teilnehmenden - ist dabei durchaus erwünscht.

\section{Erfolgsfaktoren für den Wiederbelebungsunterricht in Schulen}

1. Benennung eines schulinternen Projektkoordinators und eines Stellvertreters für den Wiederbelebungsunterricht.

1 Anmerkung zu Schritt 3 dieser Unterrichtsmethode:

Während des Schrittes 3 im Kurskonzept sollen vom Instruktor die Maßnahmen ausschließlich völlig korrekt durchgeführt werden, damit Teilnehmende auch nur den korrekten Ablauf sehen und sich diesen dann auch in dieser Weise anschaulich merken. Bei falscher oder unvollständiger Anleitung durch Kursteilnehmer soll innegehalten, gewartet und/oder nachgefragt werden, um die Möglichkeit zu bieten, die Maßnahmen korrekt anzuleiten, sich zu korrigieren und letztendlich nur Korrektes vorzuzeigen. Die Frage, welche Faktoren zum korrekten "Merken" beitragen, ist derzeit noch Gegenstand der Forschung. Sehr wahrscheinlich spielt das konkrete "Selbermachen" eine viel größere Rolle als das „Zuschauen“. Auch der Bezug zum Alltag der Teilnehmenden, in dem diese Fertigkeiten anzuwenden sind, kann zum besseren und effektiveren Lernen beitragen. Diesen Bezug soll der Ausbilder in den Übungsszenarien möglichst herstellen. 
2. Am Ende des Schulunterrichts: Einbindung einer Feedbackrunde zur abschließenden Reflektion des Wiederbelebungstrainings und Klärung offener Fragen durch die Teilnehmenden.

3. Regelmäßige Treffen der BLS-unterrichtenden Lehrerinnen und Lehrer, um sich über die Erfahrungen, Probleme und Lösungen des BLS-Unterrichts auszutauschen. Für Fragen kann der GRC jederzeit kontaktiert werden. Eine Fortbildung zum Thema mindestens alle 2 Jahre ist wünschenswert, um über den aktuellen Stand und über neue Entwicklungen in der Wiederbelebung und deren Unterricht informiert $\mathrm{zu}$ sein.

4. Vorhaltung ausreichender Übungspuppen, um die praktische Übung intensiv trainieren zu können. Alternativ: Angebot von Trainings in Kleingruppen.

5. Einbindung des Schulsanitätsdienstes.

6. Nutzung weiterer interner und externer Unterstützungsmöglichkeiten, wie z. B. Hilfsorganisationen, e-Learning Module.

\section{Lern- und Unterrichts- materialien}

\footnotetext{
Materialien, die im Rahmen des modularen GRC-Lehrerausbildungskurses ausgehändigt werden

- Kursmanuale des GRC

- GRC-BAGEH-Curriculum für die Schülerausbildung in Wiederbelebung

- Faktenblatt zu Kreislaufstillstand und Wiederbelebung

- Die 10 GRC-Prinzipien der Wiederbelebung für Laien

- Präsentation für den Wiederbelebungsunterricht in Schulen

- Vorträge des modularen GRCLehrerausbildungskurses

- Animation eines defibrillierten Herzens/Kammerflimmern

- Video zu den Basismaßnahmen zur Wiederbelebung - Innerklinische Notfallversorgung
}

- Demovideo des European Resuscitation Council (BLS-AED DemoVideo)

\section{Beispielmusik zur Einübung der Wiederbelebung mit dem richtigen Rhythmus (100-120 Beats pro Minute) \\ - Daft Punk: Get Lucky \\ - Lady GaGa: Pokerface \\ - Avicii: Wake me Up \\ - Robin Thicke: Blurred Lines \\ - Helene Fischer: Atemlos \\ - Bee Gees: Staying Alive}

\section{Links zu Filmen und Videos zur Einbindung in den Wiederbelebungsunterricht}

- European Space Agency - Video zur Wiederbelebung von der Internationalen Raumstation: https://www. youtube.com/watch?v=YVEBXvW_ LIk\&feature $=$ youtu.be

- Warum ist Wiederbelebung wichtig? Ein Prank-Video in Zusammenarbeit mit der Köln International School of Design: https://www.youtube.com/ watch?v=L8Dt4EogOjA

- Leben retten mit Herzdruckmassage - so geht es! | WDR: https://www.youtube.com/watch? $\mathrm{v}=$ BUQRtdG1uaQ

- GRC Youtube Kanal mit weiteren Videos zum Thema Reanimationsversorgung: https:// www.youtube.com/channel/ UCS2r4MeA98rum0_LXFxI$\mathrm{uA} /$ videos?disable_polymer $=1$

- Leben retten - kaum einer tut es! | odysso - Wissen im SWR: https://www.ardmediathek.de/ard/ player/Y3JpZDovL3N3ci5kZS8x ODMyNDY5MA/

- Englisches Video: Saving a life is a child's play: https://www.youtube. com/watch?v=0Yf4umHnD3c

- Zeichentrick zu Wiederbelebung für jüngere Zielgruppen: https://www.youtube.com/watch? $\mathrm{v}=\mathrm{BBBumEgCOxU}$
Aktuelle Informationen zu Reanimationsversorgung und Wiederbelebungsunterricht in Schulen finden Sie auf unserer Homepage

- www.grc-org.de

\section{Weitere deutsche Websites zu dem} Thema

- www.einlebenretten.de

- www.wiederbelebung.de

\section{Englischsprachige Website zu} europaweiten oder weltweiten Aktivitäten

- www.erc.edu: Europäischer Dachverband der Wiederbelebungsorganisationen (European Resuscitation Council)

- www.ilcor.org/wrah: Weltweiter Dachverband der Wiederbelebungsorganisationen, der die Leitlinien zur Wiederbelebung herausbringt (International Liaison Committee on Resuscitation)

- www.lifesaver.org.uk: Englischer interaktiver Lehrfilm zur Reanimation, e-Learning

- https://www.ircouncil.it/: Italian Resuscitation Council

- https://www.resus.org.uk: Resuscitation Council UK

\section{Aktionen und Veranstaltungen zum Thema Wiederbelebung}

- „Woche der Wiederbelebung“: Jedes Jahr im September findet deutschlandweit die „Woche der Wiederbelebung" statt, die auf das Thema HerzKreislauf-Stillstand und Wiederbelebung aufmerksam machen möchte. Nähere Informationen finden Sie hier: https://www.einlebenretten.de/ aktionen.html

- World Restart a Heart Day (WRAH): Seit 2018 findet jedes Jahr am 16. Oktober weltweit der World Restart a Heart Day statt. Nähere Informationen hierzu finden Sie unter: https://www.grc-org.de/ kooperationen/10-2-World-Restarta-Heart-Day \& https://www.ilcor. org/world-restart-a-heart-2019/ 


\section{Korrespondenzadresse}

Univ.-Prof. Dr. med. B. W. Böttiger

Klinik für Anästhesiologie und Operative Intensivmedizin, Universitätsklinikum Köln (AöR)

Kerpener Straße 62, 50937 Köln, Deutschland bernd.boettiger@uk-koeln.de

\section{Einhaltung ethischer Richtlinien}

Interessenkonflikt. B. Dirks, G. Rücker und H. Papaspyrou geben an, dass kein Interessenkonflikt besteht. $\mathrm{S}$. Wingen ist Vorstandsreferentin Deutscher Rat für Wiederbelebung (GRC). R. Greif ist ERC Board Director of Education and Training, ILCOR Task Force Education, Implementation, Team Chairperson, Associate Editor European Journal of Anaesthesiology, Editor-in-Chief Trends in Anaesthesia and Critical Care. B. W. Böttiger ist European Resuscitation Council (ERC) Board Director Science and Research; Vorstandsvorsitzender des Deutschen Rates für Wiederbelebung/German Resuscitation Council, (GRC); Mitglied in der "Advanced Life Support" (ALS) Task Force des International Liaison Committee on Resuscitation (ILCOR); Mitglied im Präsidium der Deutschen Interdisziplinären Vereinigung für Intensiv- und Notfallmedizin (DIVI); Schatzmeister der Deutsche Interdisziplinären Vereinigung für Intensiv und Notfallmedizin (DIVI), Landesfeuerwehrarzt und Mitglied im Beirat des Verbandes der Feuerwehren in NRW e. V., Associated Editor des European Journal of Anaesthesiology (EJA); Mitherausgeber der Zeitschrift Resuscitation; Schriftleiter der Zeitschrift Notfall + Rettungsmedizin. Für Vorträge hat er Honorare der folgenden Firmen erhalten: Medupdate $\mathrm{GmbH}$, Boehringer Ingelheim Pharma GmbH \& Co. KG, Forum für Medizinische Fortbildung (FoMF), Baxalta Deutschland GmbH, Bayer Vital GmbH, ZOLL Medical Deutschland GmbH, C. R. Bard GmbH, GS Elektromedizinische Geräte G. Stemple GmbH, Novartis Pharma $\mathrm{GmbH}$, Philips GmbH Market DACH.

Für diesen Beitrag wurden von den Autoren keine Studien an Menschen oder Tieren durchgeführt. Für die aufgeführten Studien gelten die jeweils dort angegebenen ethischen Richtlinien.

\section{Literatur}

1. European Resuscitation Council (ERC), Böttiger BW, Bossaert LL, Castrén M et al (2016) KIDS SAVE LIVES-ERC position statement on school children education in CPR: "hands that help-training children is training for life". Resuscitation 105:A1-3

\section{Gemeinsame Jahrestagung der DGIIN \& ÖGIAIN}

Kompetenz und Verantwortung

Die 51. Gemeinsame Jahrestagung der DGIIN \& ÖGIAIN findet vom 12.-14. Juni 2019 in Berlin statt und steht unter dem Motto „Kompetenz und Verantwortung". Die Förderung einer spezialisierten notfall- und intensivmedizinischen Kompetenz ist essentiell, um in diesen anspruchsvollen Bereichen eine qualitativ hochwertige Patientenversorgung zu gewährleisten. Hieraus ergibt sich die Bereitschaft, Verantwortung im Bereich der Krankenversorgung, aber auch der Lehre, Forschung und Weiterbildung zu übernehmen.

Im übergeordneten Fokus des Kongresses stehen die intensivmedizinische Versorgung in der Notaufnahme, die interprofessionelle und interdisziplinäre Zusammenarbeit sowie die Spezialisierung und Qualifikation von Ärzten und Pflegenden auf der Intensivstation und in der Notaufnahme. Ziel ist es, den Schulterschluss sowohl der verschiedenen Berufsgruppen, insbesondere der Pflege, als auch der Intensiv- und Notfallmedizin zu fördern und zu fordern.

Die medizinischen Schwerpunkte sind breit gefächert. Hier sind beispielsweise die Diskussion um die zunehmende Nutzung von extrakorporalem Life Support (ECLS), der alle Formen der Herz- bzw. Lungenunterstützung beinhaltet, und die damit eng verknüpfte Diagnostik und Therapie des Schocks sowie ethische Fragen in der Notfall- und Intensivmedizin zu nennen.

Biomarker-gesteuerte Therapien, mikrobiologisches Monitoring, multiresistente Erreger und Antibiotikatherapie sind, ebenso wie Katastrophen- und Terrorlagen, neben vielen weiteren Themen Teil des abwechslungsreichen Programms.

Zur Vertiefung einiger Themenschwerpunkte werden auch in diesem Jahr praktische Workshops angeboten. Im Sinne des letzten Konsensuspapiers zur Notfallsonographie wird dazu ein praxisnaher Kurs angeboten. Des Weiteren werden "hands on"-Kurse zu den Themen ECLS (VV/VA-ECMO; ECMO/ IMPELLA), Herzschrittmacher-Therapie, Reanimation, der schwierige Atemweg oder nicht-invasive Beatmung angeboten. Auch die Möglichkeit der Erweiterung der "soft skills" im Rahmen eines Workshops zum schwierigen Angehörigengespräch sowie ein interprofessioneller Simulations-Kurs zur Kommunikation im Rahmen des Übergabemanagements (InProSim ${ }^{\circledR}$ ) ist möglich.

Pflegebezogene Schwerpunkte sind das Schnittstellenmanagement zwischen der Präklinik, der zentralen Notaufnahme und der Intensivstation inklusive Übergabe und Ersteinschätzung. Ein weiteres wichtiges Thema ist die Spezialisierung der Notfallpflege. Auch praxisnahe Themen wie Delir-Management, NIV, Rhythmusstörungen, Zugänge und extrakorporale Verfahren kommen nicht zu kurz. Hierzu werden praktische Workshops angeboten.

Den Pflegenden bietet sich die Möglichkeit, während des gesamten Kongresses auch an anderen Veranstaltungen teilzunehmen, da die pflegerischen Beiträge kontextbezogen integriert werden, um interprofessionelles Networking zu ermöglichen.

Das Programm umfasst außerdem einen ICU-Beginner-Kurs, einen Science Slam, das Junge Forum (meet the expert, meet the guideline author, case reports, etc.) Nukleussitzungen und Industriesymposien. Für einen umfassenden Überblick über das breit gefächerte Angebot lohnt sich ein Blick in das Tagungsprogramm.

\section{9.dgiin.de}

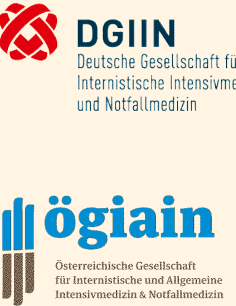

